\title{
Pulmonary arterial hypertension and chronic thromboembolic pulmonary hypertension: pathophysiology
}

\author{
M. Humbert*,\#,9
}

ABSTRACT: Pulmonary arterial hypertension (PAH) and chronic thromboembolic pulmonary hypertension (CTEPH) are two of the key subgroups of pulmonary hypertension. They are characterised by different risk factors. PAH can be associated with mutations in the gene encoding bone morphogenetic protein receptor type II (BMPR2), HIV infection, congenital heart disease, connective tissue disease (such as systemic sclerosis), and exposure to particular drugs and toxins including fenfluramine derivatives. In contrast, CTEPH can be associated with antiphospholipid antibodies, splenectomy and the presence of a ventriculo-atrial shunt or an infected pacemaker.

The first-line therapies used to treat PAH and CTEPH also differ. While medical therapy tends to be used for patients with $\mathrm{PAH}$, pulmonary endarterectomy is the treatment of choice for patients with CTEPH.

However, there are possible common mechanisms behind the two diseases, including endothelial cell dysfunction and distal pulmonary artery remodelling. Further research into these similarities is needed to assist the development of targeted pharmacological therapies for patients with inoperable CTEPH and patients who have persistent pulmonary hypertension after endarterectomy.

KEYWORDS: Chronic thromboembolic pulmonary hypertension, epidemiology, pathophysiology, pulmonary arterial hypertension

$\mathbf{T}$ he classification of pulmonary hypertension (PH) was recently updated at the 4 th World Symposium on Pulmonary Hypertension [1]. This new classification delineates six key subgroups of $\mathrm{PH}$ : pulmonary arterial hypertension (PAH); pulmonary veno-occlusive disease and/or pulmonary capillary haemangiomatosis; $\mathrm{PH}$ due to left heart disease; $\mathrm{PH}$ due to lung diseases and/or hypoxia; chronic thromboembolic pulmonary hypertension (CTEPH); and $\mathrm{PH}$ with unclear or multifactorial mechanisms. This review will focus on the pathophysiology of PAH and CTEPH.

PAH

Mechanisms of disease

When obstruction of small pulmonary arteries, which is characteristic of PAH, was first described in the 1950s [2], it was thought that vasoconstriction and thrombosis were the key mechanisms of disease. However, it is now known that vasoconstriction is the dominant feature in $<10 \%$ of patients at PAH diagnosis; these rare patients are characterised by long-term vasodilator response to calcium channel blockers [3]. Furthermore, thrombosis alone cannot explain PAH, although it may contribute to its pathogenesis. Remodelling of small pulmonary arteries $(<500 \mu \mathrm{m}$ diameter $)$ via the proliferation of smooth muscle and endothelial cells is now recognised to play a major role in the pathogenesis of $\mathrm{PAH}$ [4]. This abnormal proliferation includes hypertrophy of the media and intima, and the formation of tumour-like lesions from endothelial cells in regions of pulmonary artery bifurcation (plexiform lesions). Such proliferation is likely to be an important target for future pharmacological therapies.

\section{Risk factors}

Familial PAH accounts for $\sim 4 \%$ of PAH cases [5]. Three-quarters of these patients harbour a mutation in the gene encoding bone morphogenetic protein receptor type II (BMPR2). Patients with such mutations are described as having heritable
AFFILIATIONS

*Univ Paris-Sud, Faculté de médecine, Kremlin-Bicêtre, ${ }^{\#}$ AP-HP, Centre National de Référence de l'Hypertension Pulmonaire Sévère, Service de Pneumologie et Réanimation Respiratoire, Hôpital Antoine Béclère, and

"INSERM U999 Hypertension Artérielle Pulmonaire, Physiopathologie et Innovation Thérapeutique, Clamart, France.

CORRESPONDENCE

M. Humbert

Service de Pneumologie et

Réanimation Respiratoire

Hôpital Antoine-Béclère

Assistance Publique

Hôpitaux de Paris

Clamart

France

E-mail: marc.humbert@abc.aphp.fr

Received:

Nov 232009

Accepted after revision:

Dec 042009

PROVENANCE

Publication of this peer-reviewed article was supported by Bayer Schering Pharma AG, Germany (principal sponsor, European Respiratory Review issue 115). 
PAH, which tends to develop at a younger age than idiopathic $\mathrm{PAH}$ and often presents with a more severe clinical and haemodynamic phenotype [6]. Germline mutations in BMPR2 have also been found in $11-40 \%$ of apparently sporadic and idiopathic cases of PAH [7]. Furthermore, $10-20 \%$ of individuals affected by appetite suppressant-induced PAH carry a BMPR2 mutation $[8,9]$. The receptor encoded by BMPR2 belongs to the transforming growth factor (TGF)- $\beta$ superfamily, which plays a key role in vascular cell proliferation [10, 11]. However, the BMPR2 mutation has a penetrance of only $\sim 10-20 \%$. This suggests that this genetic predisposition increases susceptibility to PAH, but that additional risk factors need to be present to induce pulmonary vascular dysfunction at the level of endothelial cells and smooth muscle cells [12]. The role of additional risk factors is highlighted by the recently updated clinical classification of PAH [1], which emphasises that PAH can be induced by various factors, including drugs or toxins.

\section{Dysfunctional pathways and corresponding treatment options}

Several therapies have been developed for PAH, which target three key pathways (fig. 1): 1) the endothelin-1 pathway, targeted by endothelin receptor antagonists such as bosentan, sitaxsentan and ambrisentan; 2) the nitric oxide pathway, acted upon by phosphodiesterase type 5 inhibitors such as sildenafil; and 3) the prostacyclin pathway, targeted by prostacyclin analogues such as epoprostenol, treprostinil and iloprost $[3,14]$. These therapies can improve symptoms and exercise capacity. Although they have some antiproliferative properties, they do not reverse abnormal cell proliferation in vivo and, as such, do not represent a cure for PAH. Thus, growth factors such as platelet-derived growth factor, epidermal growth factor and fibroblast growth factor, which are involved in abnormal proliferation, form targets for future novel therapies. However, no convincing data have yet been published to support the use of drugs targeting these novel pathways.

\section{CTEPH}

\section{Mechanisms of disease}

Unlike PAH where vascular remodelling tends to occur in small pulmonary arteries, CTEPH is mainly associated with prominent obstructions in larger vessels. The pathophysiology of CTEPH remains unclear. The commonly accepted

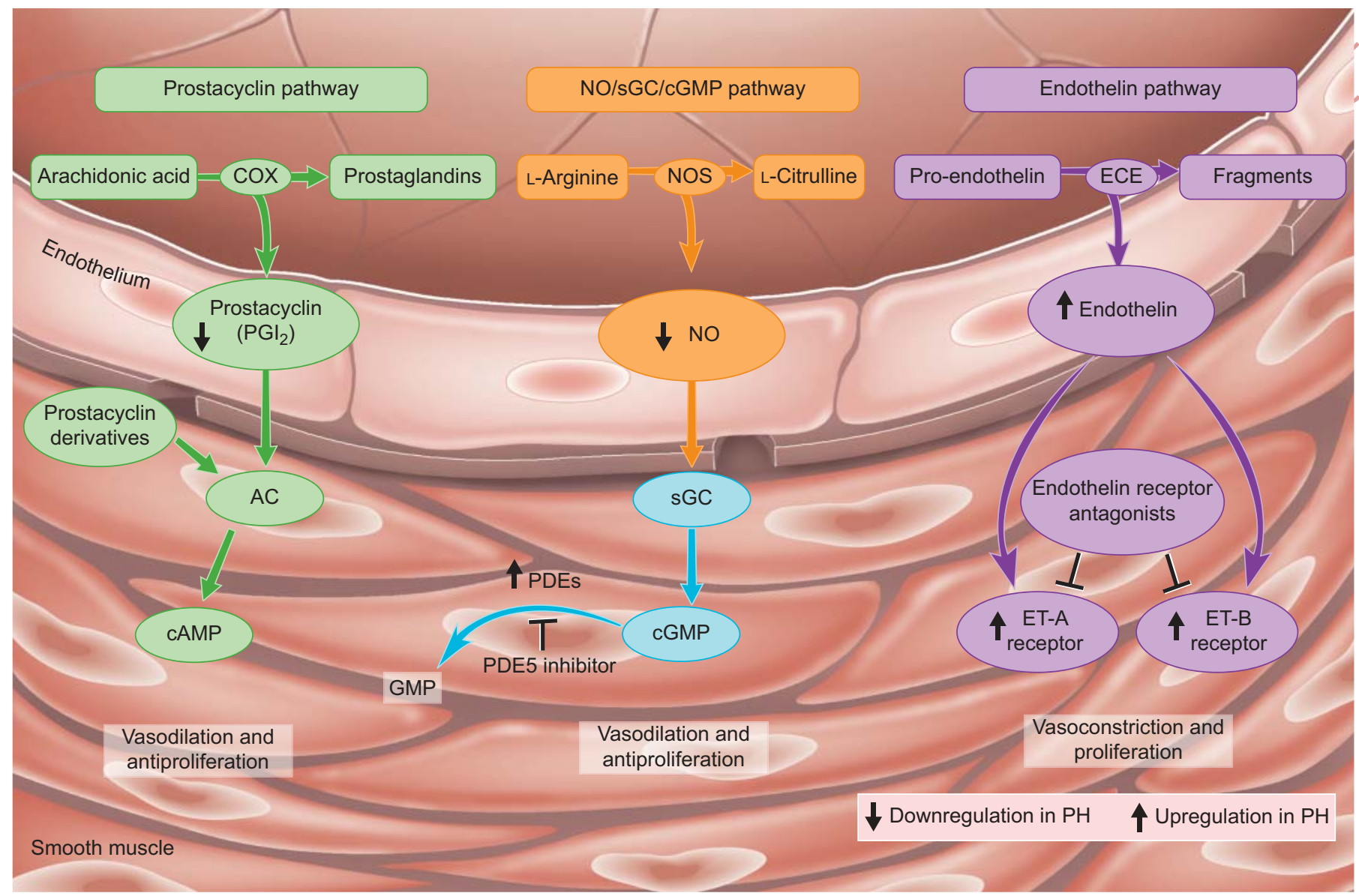

FIGURE 1. The key pathways and classes of drugs that have been approved for the treatment of pulmonary arterial hypertension. These may also have benefits in patients with chronic thromboembolic pulmonary hypertension, although further research is necessary to establish this. NO: nitric oxide; sGC: soluble guanylate cyclise; cGMP: cyclic guanosine monophosphate; COX: cyclo-oxygenase; NOS: NO synthase; ECE: endothelin converting enzyme; AC: adenylate cyclase; cAMP: cyclic adenosine monophosphate ET: endothelin; GMP: guanosine monophosphate; PDE: phosphodiesterase; PH: pulmonary hypertension. Reproduced from [13] with permission from the publisher. 


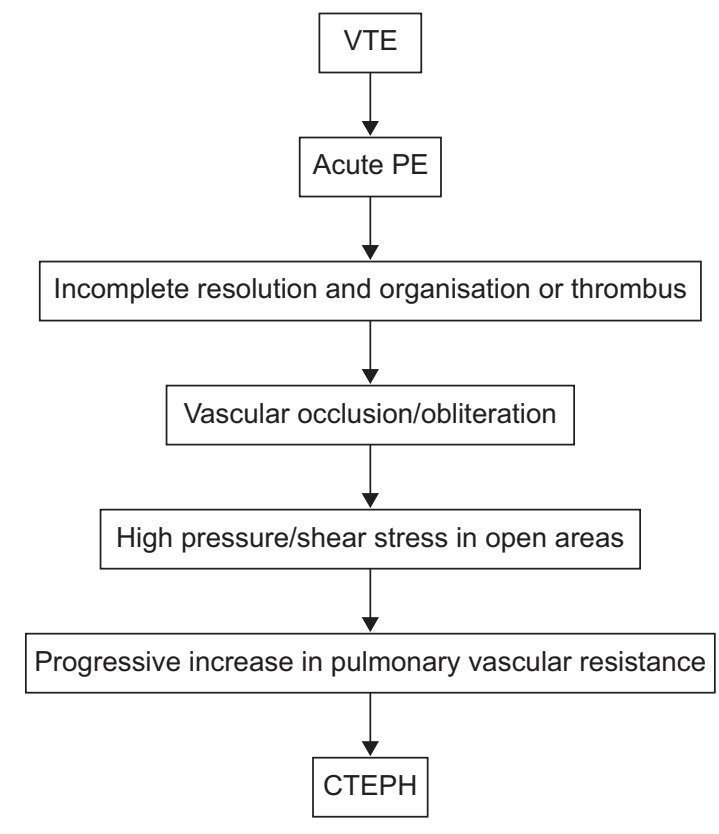

FIGURE 2. The embolic hypothesis of chronic thromboembolic pulmonary hypertension (CTEPH). VTE: venous thromboembolism; PE: pulmonary embolism.

explanation (the embolic hypothesis) is that CTEPH is the result of single or recurrent pulmonary embolism (PE) arising from sites of venous thrombosis (fig. 2) [15]. However, it has been suggested that CTEPH may also be caused by in situ thrombosis in the lung as a result of primary arteriopathy and endothelial dysfunction similar to that seen in PAH [1517]. This may help to explain why up to $63 \%$ of patients with CTEPH have no history of acute PE [18]. Interestingly, progressive remodelling may occur in small pulmonary arteries in occluded and nonoccluded territories, which supports the possible relevance of pulmonary arteriopathy in CTEPH [17].

\section{Incidence and prevalence}

Historically, the incidence of CTEPH was estimated at 0.1$0.5 \%$ in patients surviving acute PE [19]. However, a more recent study found the cumulative incidence of CTEPH to be $3.1 \%$ (95\% CI 0.7-5.5) 1 yr after PE and 3.8\% (95\% CI 1.1-6.5) 2 yrs after PE [20]. The true incidence of CTEPH after acute PE is now thought to be between $0.5 \%$ and $2 \%$ [11].

\section{Natural history}

The natural history of CTEPH is difficult to characterise because there can be a period (the so-called "honeymoon" period) of months to years between the initiating event, which may be silent, and the onset of CTEPH symptoms [21]. Furthermore, patients may initially present with nonspecific symptoms, such as mild breathlessness and a general feeling of being out of shape. The consequence of this is that patients are often only diagnosed at the late stage of the disease, when they present with the typical symptoms of progressive dyspnoea on exertion and general, clinical deterioration paralleling the loss of right ventricular functional capacity.

\section{Risk factors}

Independent predictors of CTEPH include the presence of a ventriculo-atrial shunt or infected pacemaker (OR 76, 95\% CI 8-10,351) and splenectomy (OR 18, 95\% CI 2-2,438) [22]. Chronic inflammatory disorders, such as osteomyelitis and inflammatory bowel disease are also associated with an increased risk of CTEPH (OR 67, 95\% CI 8-8,832) [23]. Previous PE (OR 19.0, 95\% CI 4.5-79.8), younger age (OR 1.8 per $10 \mathrm{yrs}, 95 \%$ CI 1.2-2.6), a larger perfusion defect (OR 2.2 per decile decrement in perfusion, 95\% CI 1.5-3.3), and idiopathic PE at presentation (OR 5.7, 95\% CI 1.4-23.0) are associated with an increased risk of CTEPH after acute PE [20].

Anti-phospholipid antibodies are the most prevalent biological abnormality found in patients with CTEPH. Indeed, antiphospholipid antibodies are more common in patients with CTEPH than in patients with PAH [24]. In addition, recent reports indicate that significantly higher levels of plasma factor VIII have been found in patients with CTEPH than in healthy controls $(41 \%$ versus $5 \%$; $p<0.0001)$ [25]. This study also found plasma factor VIII levels to be significantly higher in patients with CTEPH than in patients with PAH ( $41 \%$ versus $22 \%$; $\mathrm{p}<0.022)$. There is, however, no evidence of a link between the risk of CTEPH and antithrombin deficiency, protein C deficiency, protein S deficiency or factor V Leiden [24].

An abnormal fibrinolytic response may also help to explain why some patients develop CTEPH after an acute PE. One study of genetic mutations underlying fibrinogen structural variants reported a prevalence of dysfibrinogenaemia of $15 \%$ (95\% CI 3-27) in 22 patients with CTEPH [26]. However, it should be noted that the prevalence of dysfibrinogenaemia in the general population is unknown.

\section{Treatment options}

On multidetector helical computed tomography of the chest or on pulmonary angiography, acute PE appears as a floating clot in the pulmonary artery surrounded by contrast. Therefore, the clots are very simple to remove if necessary. CTEPH, however, appears as abnormal thickening of the pulmonary arterial wall and is characterised by the presence of fibrous scars that occlude the pulmonary artery lumen. It should also be noted that the bronchial arteries are often markedly enlarged in CTEPH due to bronchial arterial angiogenesis. Pulmonary endarterectomy is associated with excellent results [27] and is the treatment of choice for patients with CTEPH [28]. However, there are risks associated with pulmonary endarterectomy, and CTEPH is inoperable in at least $20-40 \%$ of patients [29] because of distal disease or comorbidities [27].

Therefore, there is a need for effective pharmacotherapies for the treatment of CTEPH [30]. PAH therapy is sometimes considered as a bridge to surgery in patients with severe but accessible CTEPH [31]. PAH drugs belonging to the three traditional classes have been studied in CTEPH [32-34], and novel drugs that tackle vasoconstriction and proliferation [30, $35,36]$, as well as therapies based on growth factors, may also have benefits in patients with CTEPH. However, no drug is currently approved for the medical management of CTEPH and further studies are needed to establish the efficacy of these agents in that setting [32]. 


\section{CONCLUSIONS}

PAH and CTEPH are characterised by different risk factors. $\mathrm{PAH}$ can be associated with mutations in the BMPR2 gene, HIV infection, congenital heart disease, connective tissue disease (such as systemic sclerosis) and exposure to particular drugs and toxins including fenfluramine derivatives. In contrast, CTEPH can be associated with anti-phospholipid antibodies, splenectomy and the presence of a ventriculo-atrial shunt or an infected pacemaker. The first-line therapies that are used to treat PAH and CTEPH are also different. While medical therapy tends to be used in patients with $\mathrm{PAH}$, pulmonary endarterectomy is the treatment of choice in patients with CTEPH. However, there are possible common mechanisms behind the two diseases, including endothelial cell dysfunction and distal pulmonary artery remodelling. Further research into these similarities will aid the development of targeted therapies for patients with inoperable CTEPH and patients who have persistent pulmonary hypertension after endarterectomy.

\section{STATEMENT OF INTEREST}

M. Humbert has relationships with drug companies including Actelion, Bayer Schering, GlaxoSmithKline, Novartis, Pfizer and United Therapeutics. In addition to being investigator in trials involving these companies, relationships include consultancy service and membership of scientific advisory boards.

\section{ACKNOWLEDGEMENTS}

Medical writing support was provided by C. Hill (Oxford PharmaGenesis Ltd, Oxford, UK) on behalf of Bayer Schering Pharma AG (Berlin, Germany). This article is based on a presentation given at a symposium supported by Bayer Schering Pharma AG at the 2009 European Society of Cardiology meeting held in Barcelona, Spain.

\section{REFERENCES}

1 Simonneau G, Robbins IM, Beghetti M, et al. Updated clinical classification of pulmonary hypertension. J Am Coll Cardiol 2009; 54: Suppl. 1, S43-S54.

2 Newman JH. Pulmonary hypertension. Am J Respir Crit Care Med 2005; 172: 1072-1077.

3 Humbert M, Sitbon O, Simonneau G. Treatment of pulmonary arterial hypertension. $N$ Engl J Med 2004; 351: 1425-1436.

4 Tuder RM, Abman SH, Braun T, et al. Development and pathology of pulmonary hypertension. J Am Coll Cardiol 2009; 54: Suppl. 1, S3-S9.

5 Humbert M, Sitbon O, Chaouat A, et al. Pulmonary arterial hypertension in France: results from a national registry. Am J Respir Crit Care Med 2006; 173: 1023-1030.

6 Souza R, Jardim C. Trends in pulmonary arterial hypertension. Eur Respir Rev 2009; 18: 7-12.

7 Davies RJ, Morrell NW. Molecular mechanisms of pulmonary arterial hypertension: role of mutations in the bone morphogenetic protein type II receptor. Chest 2008; 134: 1271-1277.

8 Humbert M, Deng Z, Simonneau G, et al. BMPR2 germline mutations in pulmonary hypertension associated with fenfluramine derivatives. Eur Respir J 2002; 20: 518-523.

9 Souza R, Humbert M, Sztrymf B, et al. Pulmonary arterial hypertension associated with fenfluramine exposure: report of 109 cases. Eur Respir J 2008; 31: 343-348.

10 Thomson JR, Trembath RC. Primary pulmonary hypertension: the pressure rises for a gene. J Clin Pathol 2000; 53: 899-903.

11 Galie N, Hoeper MM, Humbert M, et al. Guidelines for the diagnosis and treatment of pulmonary hypertension: The Task
Force for the Diagnosis and Treatment of Pulmonary Hypertension of the European Society of Cardiology (ESC) and the European Respiratory Society (ERS), endorsed by the International Society of Heart and Lung Transplantation (ISHLT). Eur Heart J 2009; 30: 2493-2537.

12 Humbert M. More pressure on pulmonary hypertension. Eur Respir Rev 2009; 18: 1-3.

13 Ghofrani HA, Grimminger F. Modulating cGMP to treat lung diseases. Handb Exp Pharmacol 2009; 191: 469-483.

14 Galie N, Negro L, Simonneau G. The use of combination therapy in pulmonary arterial hypertension: new developments. Eur Respir Rev 2009; 18: 148-153.

15 Peacock A, Simonneau G, Rubin L. Controversies, uncertainties and future research on the treatment of chronic thromboembolic pulmonary hypertension. Proc Am Thorac Soc 2006; 3: 608-614.

16 Egermayer P, Peacock AJ. Is pulmonary embolism a common cause of chronic pulmonary hypertension? Limitations of the embolic hypothesis. Eur Respir J 2000; 15: 440-448.

17 Moser KM, Bloor CM. Pulmonary vascular lesions occurring in patients with chronic major vessel thromboembolic pulmonary hypertension. Chest 1993; 103: 685-692.

18 Hoeper MM, Mayer E, Simonneau G, et al. Chronic thromboembolic pulmonary hypertension. Circulation 2006; 113: 2011-2020.

19 Fedullo PF, Auger WR, Kerr KM, et al. Chronic thromboembolic pulmonary hypertension. N Engl J Med 2001; 345: 1465-1472.

20 Pengo V, Lensing AW, Prins $\mathrm{MH}$, et al. Incidence of chronic thromboembolic pulmonary hypertension after pulmonary embolism. N Engl J Med 2004; 350: 2257-2264.

21 Manecke GR Jr, Wilson WC, Auger WR, et al. Chronic thromboembolic pulmonary hypertension and pulmonary thromboendarterectomy. Semin Cardiothorac Vasc Anesth 2005; 9: 189-204.

22 Bonderman D, Wilkens H, Wakounig S, et al. Risk factors for chronic thromboembolic pulmonary hypertension. Eur Respir J 2009; 33: 325-331.

23 Bonderman D, Jakowitsch J, Adlbrecht C, et al. Medical conditions increasing the risk of chronic thromboembolic pulmonary hypertension. Thromb Haemost 2005; 93: 512-516.

24 Wolf M, Boyer-Neumann C, Parent F, et al. Thrombotic risk factors in pulmonary hypertension. Eur Respir J 2000; 15: 395-399.

25 Bonderman D, Turecek PL, Jakowitsch J, et al. High prevalence of elevated clotting factor VIII in chronic thromboembolic pulmonary hypertension. Thromb Haemost 2003; 90: 372-376.

26 Morris TA, Marsh JJ, Chiles PG, et al. High prevalence of rare fibrinogen mutations in chronic thromboembolic pulmonary hypertension. Blood 2009; 114: 1929-1936.

27 Lang IM. Managing chronic thromboembolic pulmonary hypertension: pharmacological treatment options. Eur Respir Rev 2009; 18: 24-28.

28 Auger WR, Fedullo PF. Chronic thromboembolic pulmonary hypertension. Semin Respir Crit Care Med 2009; 30: 471-483.

29 Mayer E. Surgical and post-operative treatment of chronic thromboembolic pulmonary hypertension. Eur Respir Rev 2010; 19: 64-67.

30 Kim NH. Riociguat: an upcoming therapeutic tool in chronic thromboembolic pulmonary hypertension? Eur Respir Rev 2010; 19: 68-71.

31 Jensen KW, Kerr KM, Fedullo PF, et al. Pulmonary hypertensive medical therapy in chronic thromboembolic pulmonary hypertension before pulmonary thromboendarterectomy. Circulation 2009; 120 1248-1254

32 Jais X, D'Armini AM, Jansa P, et al. Bosentan for treatment of inoperable chronic thromboembolic pulmonary hypertension: BENEFiT (Bosentan Effects in iNopErable Forms of chronIc Thromboembolic pulmonary hypertension), a randomized, placebo-controlled trial. J Am Coll Cardiol 2008; 52: 2127-2134.

33 Ghofrani HA, Schermuly RT, Rose F, et al. Sildenafil for longterm treatment of nonoperable chronic thromboembolic pulmonary hypertension. Am J Respir Crit Care Med 2003; 167: $1139-1141$. 
34 Cabrol S, Souza R, Jais X, et al. Intravenous epoprostenol in inoperable chronic thromboembolic pulmonary hypertension. J Heart Lung Transplant 2007; 26: 357-362.

35 Ghofrani HA, Hoeper MM, Hoeffken G, et al. Riociguat dose titration in patients with chronic thromboembolic pulmonary hypertension (CTEPH) or pulmonary arterial hypertension (PAH) Am J Respir Crit Care Med 2009; 179: A3337.

36 Ghofrani HA, Grimminger F. Soluble guanylate cyclase stimulation: an emerging option in pulmonary hypertension therapy. Eur Respir Rev 2009; 18: 35-41. 